\title{
VENDRE SES ORGANES : UN CAS DE PRÉJUDICE CONSENTI ? Alicia-Dorothy Mornington
}

Presses de Sciences Po (P.F.N.S.P.)| « Raisons politiques »

$2011 / 4 n^{\circ} 44 \mid$ pages 57 à 77

ISSN 1291-1941

ISBN 978272463422

Article disponible en ligne à l'adresse :

http://www.cairn.info/revue-raisons-politiques-2011-4-page-57.htm

\section{Pour citer cet article :}

Alicia-Dorothy Mornington, "Vendre ses organes : un cas de préjudice consenti ? 》, Raisons politiques 2011/4 ( $\left.\mathrm{n}^{\circ} 44\right)$, p. 57-77.

DOI 10.3917/rai.044.0057

Distribution électronique Cairn.info pour Presses de Sciences Po (P.F.N.S.P.).

(C) Presses de Sciences Po (P.F.N.S.P.). Tous droits réservés pour tous pays.

La reproduction ou représentation de cet article, notamment par photocopie, n'est autorisée que dans les limites des conditions générales d'utilisation du site ou, le cas échéant, des conditions générales de la licence souscrite par votre établissement. Toute autre reproduction ou représentation, en tout ou partie, sous quelque forme et de quelque manière que ce soit, est interdite sauf accord préalable et écrit de l'éditeur, en dehors des cas prévus par la législation en vigueur en France. Il est précisé que son stockage dans une base de données est également interdit. 


\section{ALICIA-Dorothy Mornington}

\section{Vendre ses organes : un cas de préjudice consenti ?}

50 DOLLARS : VOILÀ LA SOMME pour laquelle on
peut acheter son rein à un Irakien ${ }^{1}$. Le commerce
d'organes, illégal dans la plupart des États du globe $^{2}$, est un marché lucratif, pratiqué dans plus de quatre-vingts pays $^{3}$. S'il est difficile de connaître le montant exact des transactions, l'UNICEF estimait en 2004 que ce trafic générait environ douze milliards de dollars de bénéfices annuels ${ }^{4}$. Vendre ses organes

1. Jeff Whitehead, "The Harvest: Human Organs and Human Security", Human Security Journal, printemps 2008, vol. 6, p. 20.

2. Le seul pays à ce jour ayant légalisé la vente d'organes est l'Iran, qui le fit en 1988. Pour plus d'information sur ce sujet, voir Benjamin E. Hippen, "Organ Sales and Moral Travails, Lessons from the Living Kidney Vendor Program in Iran ", Policy Analysis, $\mathrm{n}^{\circ}$ 614, mars 2008, et Ali Nobakht Haghighi et N. Ghahramani, "Living Unrelated Kidney Donor Transplantation in Iran ", Nature Clinical Practice Nephrology, vol. 2, 2006. Il existe par contre de nombreux pays n'ayant pas de législation à ce sujet, comme la plupart des pays africains par exemple.

3. Yosuke Shimazono, "The State of International Organ Trade : a Provisional Picture Based on Integration of Available Information ", Bulletin of the World Health Organization, décembre 2007 , vol. $85, \mathrm{n}^{\circ} 12$, p. $955-962$.

4. Les prix sur le marché noir sont difficiles à connaître avec exactitude, mais on suppose que selon les pays, un rein par exemple peut valoir de 750 dollars en Irak jusqu’à plus de 7500 dollars en Turquie. J. Whitehead, "The Harvest: Human Organs and Human Security ", art. cité, p. 19. Cet écart reflète le niveau de vie : plus le pays d'origine du vendeur est pauvre, moins son organe est cher, et quant aux vendeurs de pays développés, certes bien moins représentatifs du trafic mondial, ils vendent leurs organes pour des sommes beaucoup plus élevées. En somme, cette constatation de la différence de prix est comparable à la différence de prix du travail entre pays riches et pauvres.

Raisons politiques, $\mathrm{n}^{\circ} 44$, novembre 2011, p. 57-78.

(C) 2011 Presses de Sciences Po. 
est devenu une pratique courante dans certains pays en voie de développement ${ }^{5}$, tout particulièrement dans certains États de l'Inde $^{6}$. En s'y promenant, il n'est pas rare de voir des personnes portant une cicatrice au flanc, signe d'un prélèvement de rein. Si le rein est l'organe le plus vendu, il existe aussi un trafic de cornées, de lobes de foie, etc. En règle générale, l'offre suivant la demande, on peut trouver sur le marché noir n'importe quelle partie du corps humain ${ }^{7}$.

Ce marché est le lieu de rencontre de deux détresses, celle du vendeur et celle de l'acheteur. Dressons le portrait du premier : souvent illettré et endetté ${ }^{8}$, il est généralement issu d'un pays en développement. La vente s'impose à lui comme ultime recours : il est généralement contraint par ses proches ou ses créanciers à vendre un rein pour obtenir de l'argent rapidement ${ }^{9}$. La situation de l'acheteur est tout aussi urgente : issu d'un pays développé, il est malade et mourra sans doute dans l'année qui suit car la liste d'attente sur laquelle il est inscrit ne lui permettra probablement pas d'être greffé à temps ${ }^{10}$. Il se résout donc à acheter un organe sur le marché noir. Les maux de ces deux protagonistes ont des causes différentes, ils partagent pourtant une volonté acharnée de survivre. Leurs situations sont toutes deux dramatiques, puisque la contrainte les pousse à participer à un échange auquel ils répugneraient en temps normal.

5. Y. Shimazono, "The State of International Organ Trade... ", art. cité, p. 957.

6. Les pays les plus touchés sont l'Inde, le Pakistan, les Philippines, certains pays de l'Europe de l'Est, de l'Afrique sub-saharienne, mais on trouve aussi exceptionnellement des vendeurs d'organes parmi les franges les plus pauvres de pays développés, tels que les États-Unis ou l'Espagne. Voir Nancy Schepper-Hughes, "The Ultimate Commodity ", The Lancet, octobre 2005, vol. 366, p. 1349-1350.

7. Pour la description détaillée de parties du corps que l'on peut greffer, voir Andrew Kimbrell, The Human Body Shop, New York, Harper Collins, 1993, p. 30. Il est aussi possible de se procurer des corps entiers comme l'a montré le cas de l'exposition "Our Body " où la justice française a suspecté que des corps de prisonniers chinois avaient été obtenus de manière illégale pour être ensuite disséqués, enduits de résine et exposés à travers le monde. Voir "La justice interdit l'exposition "Our Body" ", LeMonde.fr, 21 avril 2009, http://www.lemonde.fr/culture/article/2009/04/21/la-justice-interdit-lexposition-our-body_1183328_3246.html.

8. J. Whitehead, "The Harvest: Human Organs and Human Security ", art. cité, p. 24. Voir aussi le Bulletin de l'Organisation Mondiale de la Santé, vol. 82, $\mathrm{n}^{\circ}$ 9, septembre 2004, p. 639-718.

9. N. Schepper-Hughes, "The Ultimate Commodity", art. cité, p. 195.

10. Cette pénurie de greffes s'explique par plusieurs causes: 1) la réticence des familles à accepter le don d'organes de leur proche décédé ; 2) l'augmentation de maladies dans le monde développé nécessitant une greffe (la greffe de rein a pour cause première l'obésité et le surpoids car ils entraînent très souvent un diabète de type 2 , qui conduit généralement à l'insuffisance rénale). 
Leur rencontre se déroule généralement sur Internet. Il existe en effet de nombreux sites spécialisés, tels que Findonors.com. Des annonces furent publiées aussi sur Ebay, avant d'être retirées par les administrateurs du site ${ }^{11}$. Ce type d'échange n'est pas nouveau : on peut voir, dans Les Misérables, Fantine acculée par les dettes à vendre ses cheveux puis ses dents afin de sauver sa fille de la misère ${ }^{12}$. Mais la mondialisation des moyens de transport et de communication a considérablement augmenté l'échelle de ce type de marché, devenu un phénomène global depuis les années 1980 et qui ne cesse de croître ${ }^{13}$.

Cette évolution est aussi liée aux progrès de la technique de la greffe. Les avancées chirurgicales et l'amélioration de l'efficacité thérapeutique des médicaments anti-rejet donnent aujourd'hui aux greffés une espérance de vie quasi-normale. L'espoir des malades de voir leur vie prolongée s'est mué en revendication d'un "droit " à la greffe et la littérature sur ce sujet fait écho à cette exigence ${ }^{14}$. Les organes disponibles étant une ressource limitée, de nombreux patients meurent en attente d'une greffe. Aux États-Unis, en 2009, 26000 personnes sur liste d'attente sont décédées alors qu'elles auraient probablement pu être sauvées par une greffe de rein ${ }^{15}$. L'Agence française de biomédecine déclare qu' " environ 30 malades sont inscrits sur la liste d'attente par jour ouvré ${ }^{16} »$. Le Conseil de l'Europe notait en 2003 que 15 à $30 \%$ des malades inscrits sur liste d'attente n'obtiennent pas de greffe à temps ${ }^{17}$.

11. Mark Schweda et Silke Schicktanz, "The "Spare Parts Person" ? Conceptions of the Human Body and their Implications for Public Attitudes Towards Organ Donation and Organ Sale", Philosophy, Ethics, and Humanities in Medicine, vol. 4, n 4, 2009, p. 1.

12. Victor Hugo, Les Misérables, Paris, Garnier Flammarion, 1967, éd. par Renée Journet, t. 1 , p. 215.

13. Y. Shimazono, "The State of International Organ Trade... ", art. cité.

14. Voir par exemple Cécile Fabre, "Justice and the Coercive Taking of Cadaveric Organs ", British Journal of.Political Science, vol. 34, 2004, p. 69-86. L'auteure défend l'existence d'un "droit " d'obtenir une greffe cadavérique.

15. J. Whitehead, "The Harvest : Human Organs and Human Security ", art. cité, p. 19.

16. Agence de la biomédecine. 2010. Rapport d'activité de l'Agence de la biomédecine. Disponible en ligne: http://www.agence-biomedecine.fr/uploads/document/RA_ BIOMED_2010_Internet.pdf, p. 22.

17. Conseil de l'Europe, rapporteur : Mrs Ruth-Gaby Vermot-Mangold, "Trafficking in Organs in Europe ", Report, Social, Health and Family Affairs Committee, Suisse, SOC, document 9822, 3 juin 2003, disponible en ligne: http://assembly.coe.int/ documents/workingdocs/doc03/edoc9822.htm. Présentés ainsi, ces chiffres suggèrent que ces personnes sont décédées faute de greffe, alors qu'en réalité, elles sont mortes des suites de leur maladie. Je remercie une infirmière élève du Diplôme universitaire 
Depuis quelques années, des voix s'élèvent pour dénoncer la pénurie d'organes et proposent d'y remédier en légalisant le marché noir. Les partisans de cette solution se servent en général de trois types d'arguments. Le premier est réaliste: interdire de jure un marché qui existe de facto n'est pas efficace. Le second est conséquentialiste : légaliser permettrait de sauver la vie de malades en attente de greffe ${ }^{18}$ et d'améliorer celle des vendeurs qui pourraient alors être rémunérés plus justement. Enfin, le troisième argument est déontologique. Il s'appuie sur l'interprétation libertarienne ${ }^{19}$ de la propriété de soi, selon laquelle le corps est la propriété absolue de l'individu. Toute limitation hétéronome à la libre jouissance de cette propriété est donc une injustice. Dans les pays anglo-saxons, cet argumentaire gagne en légitimité dans les débats médicaux, juridiques, académiques et politiques, comme le démontrent les chercheurs américains Sheila et David Rothman ${ }^{20}$. Certains pays, comme la France, se montrent beaucoup plus réticents. S’il existe certaines exceptions, dans leur grande majorité, chercheurs, politiciens et praticiens rejettent ce type discours.

Aux sources du problème de la vente d'organes se trouve une personne qui consent à vendre une partie de son corps. C'est sur elle que portera notre analyse. En effet, en étudiant la nature du consentement du vendeur, nous voulons nous placer au cœur du débat éthique et politique sur la vente d'organes. Ce consentement pose de nombreuses difficultés. En droit, la vente d'organes est assimilée à un cas de " préjudice consenti ${ }^{21}$ ". Ce terme désigne une

d'éthique de l'hôpital Saint Anne à Paris, rencontrée le 19 mai 2010, d'avoir souligné ce point.

18. Notons que cette prémisse souvent mobilisée par les défenseurs de la légalisation n'est valable qu'au sujet de la vente d'organes vitaux. Or, il existe un marché d'organes non vitaux, comme celui de la cornée par exemple, qui est assez répandu; et dans ce cas, le vendeur de cornée améliore le confort du malade en lui permettant de voir à nouveau. Il ne lui sauve pas la vie, mais lui redonne la vue au prix de la sienne.

19. Nous faisons ici référence au libertarianisme classique, dit « de droite » depuis l'apparition du libertarianisme dit "de gauche». À ce sujet, voir: Michael Otsuka, "Comment être libertarien sans être inégalitaire", Raisons Politiques, $\mathrm{n}^{\circ} 23$, août 2006, p. 9-22.

20. Sheila Rothman et David Rothman, "The Hidden Cost of Organ Sale ", The American Journal of Transplantation, vol. 6, 2006, p. 1524. Pour un résumé des prises de position dans la littérature académique internationale, voir Valérie Gateau, Pour une philosophie du don d'organes, Paris, Vrin, 2009, p. 72-74.

21. C'est la traduction que nous donnons au terme harm to self de Joel Feinberg, pionnier dans l'étude systématique de ces cas limites. Voir Joel Feinberg, The Moral Limits of the Criminal Law. Vol. 3: Harm to Self, Oxford, Oxford University Press, 1986. 
situation dans laquelle un individu se fait du tort à lui-même, ou accepte qu'on lui en fasse, de manière volontaire et informée. Mais ce concept de préjudice consenti est problématique puisqu'il sousentend une vision substantielle du bien. En d'autres termes, il ignore la motivation du sujet et l'appréciation qu'il fait de son propre choix. L'exemple classique est celui du masochiste qui ne pense pas nécessairement la douleur comme "préjudice ", puisque, par définition, elle lui est agréable ou du moins désirable. De même, le vendeur ne voit pas nécessairement la vente d'un rein comme un préjudice puisqu'elle lui permet d'obtenir de l'argent rapidement. On pourrait donc s'étonner qu'en droit ces cas soient décrits comme "consentement de la victime". De quoi ou de qui seraient-ils les victimes? En pratique, cet étonnement se heurte à un principe de réalité : dans un système juridique rationnel, la détermination du préjudice ne peut être laissée à l'appréciation de chacun. Pour être efficace, le droit pénal doit fournir une définition objective du préjudice et donc s'appuyer sur une vision substantielle du bien. De ce fait, si le droit devient neutre, il disparaît, et l'État avec lui, se voyant réduit dans le meilleur des cas à une "minarchie".

Il existe pourtant une toute autre lecture du préjudice consenti. Elle s'articule autour de la maxime de droit romain volenti non fit injuria, qui signifie littéralement que l'on ne peut pâtir de ce à quoi l'on consent. On lit en général cette formule comme une contestation de l'absence de neutralité du droit. Cette maxime est en réalité plus complexe. Nous avons choisi de nous y attarder car elle rassemble toutes les dimensions du problème du préjudice consenti tout en permettant d'en déployer les enjeux ${ }^{22}$. Elle peut être lue de manière descriptive $a$ ), ou nominative $b^{\prime}$ ) et $b^{\prime \prime}$ ).

a) De manière purement descriptive, la maxime se lit ainsi : si A consent à $\mathrm{X}, \mathrm{X}$ ne peut porter préjudice à $\mathrm{A}$. Le consentement annulerait la nature préjudiciable d'un acte, qui le serait en toute autre circonstance. Cette maxime est alors conforme ou non au réel. Par conséquent, si l'on identifie un seul cas où une personne est préjudiciée par ce à quoi elle consent, alors cette maxime est fausse. Cette interprétation laisse à désirer, puisque les termes ne sont pas définis précisément. Il faut alors envisager une autre lecture : b) la maxime est nominaliste, c'est-à-dire que son sens dépend de celui attribué aux termes volenti et injuria. Ces mots sont

22. Je suis profondément reconnaissante envers Christian List pour ces remarques. 
polysémiques: en latin, la volentia signifie consentement ou volonté $^{23}$ et l'injuria recouvre une injustice, un tort ou un dommage $^{24}$. Afin de clarifier le propos, nous comprenons volenti comme consentement et injuria comme préjudice.

Cela conduit à deux possibilités :

b') L'existence du volenti est tangible. Elle exclut alors la possibilité de l'injuria. La maxime se lit alors de la sorte : étant donné que $\mathrm{A}$ consent à $\mathrm{X}, \mathrm{X}$ ne porte pas préjudice à $\mathrm{A}$. En d'autres termes, le concept de préjudice consenti serait donc contradictoire puisque, par définition, l'objet du consentement serait soit un bien, soit un acte non-préjudiciable. Ce qui pourrait sembler préjudiciable à un tiers ne constituerait donc pas une source de préjudice pour l'individu consentant. Si une personne consent à vendre un rein, alors pour elle ce choix sera nécessairement un bien ou du moins un acte non-préjudiciable.

Ou b") l'injuria est vérifiée. Par conséquent, l'existence d'injuria exclut la possibilité du volenti. On considère alors que : étant donné que X est préjudiciable, A ne peut consentir à X. Si l'on pose que la vente d'organes constitue un préjudice réel aux vendeurs d'organes, alors leur consentement ne peut être sincère ou réel. Le vendeur peut donner l'apparence du consentement, pourtant, selon cette lecture, il ne consentirait pas réellement. Il faut ici distinguer plusieurs niveaux de consentement: le consentement manifeste et le consentement hypothétique. Un individu signe un contrat par lequel il vend son rein - il s'agit là de son consentement manifeste. Cela ne correspond peut-être pas à son consentement hypothétique, si l'on considère qu'une personne rationnelle ne souhaiterait pas vendre de rein.

Pour comprendre la notion de préjudice consenti dans le cas de la vente d'organes, nous envisagerons ces deux facettes de la maxime volenti non fit injuria, en commençant par voir si l'on trouve chez les vendeurs de la volentia (I), puis nous poserons la question de l'injuria (II). Cette démarche exclut donc nécessairement une discussion sur les répercussions sociales du commerce d'organes, puisqu'elle se centre sur l'individu qui consent à vendre.

23. Félix Gaffiot, Dictionnaire Latin-Français, Paris, Édition Hachette, 1934, p. 1690.

24. Ibid., p. 822. 
Afin d'évaluer la nature du consentement des vendeurs d'organes, il nous faut avant tout définir ce que nous entendons par consentement. Il s'agit d'un acte par lequel on donne son assentiment. Comme nous allons le voir, le consentement a une importance capitale pour les libéraux, mais aussi pour les juristes, puisqu'il est la clef de voûte du contrat.

Au cour de la pensée libérale, le consentement individuel est un concept aussi séduisant que trompeur. Il représente la force émancipatrice par laquelle l'homme de la Renaissance passe du statut de sujet inféodé à son monarque à celui d'individu libre et rationnel. La volonté de ce dernier devient, à partir du Léviathan de Hobbes, le critère central de la légitimité de l'État. Son autorité fondée sur le consentement, l'État n'a plus besoin d'imposer à l'individu son joug par la force puisque ce dernier y consent de lui-même. Le consentement est donc une fiction essentielle de la pensée libérale et est synonyme de soumission au pouvoir. Un siècle plus tôt, La Boétie dénonçait cela en disant, au sujet de l'absolutisme : " c'est le peuple qui s'asservit, qui se coupe la gorge, qui, ayant le choix ou d'être serf ou d'être libre, quitte la franchise et prend le joug, qui consent à son $\mathrm{mal}^{25}$ ". L'anthropologie libérale est flatteuse pour l'individu, décrit comme rationnel, autonome, et l'égal en droit des puissants. Dans toutes les théories libérales contractualistes, c'est l'État qui est identifié comme la solution la plus avantageuse pour l'individu. Le consentement, expression de la liberté et de l'autonomie individuelle, ne semble servir qu'à justifier la domination. Même chez certains libertariens comme Robert Nozick, la liberté individuelle conduit à rejeter l'anarchie et à défendre l'existence un État, aussi minimal soit-il ${ }^{26}$.

Cette fiction du consentement et de la rationalité individuelle est à la source d'une autre soumission, celle présente dans le contrat. Pour donner l'exemple du droit français, comparable sur ce point à tout autre ordre juridique, le consentement est défini comme un accord de volontés générateur de droits et d'obligations entre les

25. Étienne de La Boétie, Discours de la servitude volontaire, Paris, Garnier Flammarion, 1983, p. 136 (nous soulignons).

26. Robert Nozick, Anarchy, State, and Utopia, Oxford, Blackwell, 1974, préface, p. ix. Il veut " un État minimal, limité aux seules fonctions de protection contre la violence, le vol, la fraude, et garantissant le respect des contrats ", (notre traduction). 
parties qui le concluent ${ }^{27}$. Pour qu'un contrat soit valable, il faut qu'il soit fondé sur le consentement libre, réciproque et éclairé des parties contractantes ${ }^{28}$. Par ailleurs, leur consentement peut être invalidé par manque d'information - on parle de «vice du consentement". On distingue trois catégories principales rendant un contrat nul : l'erreur, le dol et la violence ${ }^{29}$. Cette conception du contrat comme accord de volontés fait entièrement abstraction du rapport de force qui peut exister entre les contractants, en les présumant égaux et capables d'émettre un consentement libre, alors que l'on peut évidemment consentir à contrecœur - il s'agit d'ailleurs de la différence entre consentement et volonté. Par exemple, on consent à s'acquitter d'une dette sans nécessairement avoir un franc désir de le faire toutes choses égales par ailleurs ${ }^{30}$. C'est pour cela que notre maxime romaine prend tout son sens dès lors que l'on traduit volenti par "consentement " et non par "volonté ».

Si le consentement est une condition nécessaire pour établir un contrat, il ne constitue pas pour autant une condition suffisante. Le Code civil français, par exemple, estime que la cause (l'objet) du contrat : "est illicite, quand elle est prohibée par la loi ${ }^{31}$ ". La vente d'organes est interdite en l'occurrence parce qu'elle porte sur un objet indisponible au commerce : le corps humain. Le consentement (volenti) de l'individu à vendre un organe n'est donc absolument pas pertinent ici puisque, par nature, cet acte ne peut être en aucun cas autorisé. L'interdiction de la vente d'organes constitue ainsi un parfait exemple de paternalisme. On appelle paternalisme une intervention plus ou moins coercitive de l'État dans la sphère

27. Jean-Luc Aubert, Le contrat, droit des obligations, Paris, Dalloz, 2005 [3 éd.], p. 5. Le consentement est nécessaire dans les pays de common law aussi bien que de droit civil. On évoque la nécessité d'obtenir l'accord ou le consentement mutuel des parties contractantes (a meeting of minds).

28. Pour la France, voir les articles de 1109 à 1119 du Code civil. Disponible en ligne sur le site Legifrance :

http://www.legifrance.gouv.fr/affichCode.do;jsessionid=73B64820D7B9B150B8F6 AF0D13B0664F.tpdjo02v_3?idSectionTA=LEGISCTA000006150237\&cidTexte= LEGITEXT000006070721\&dateTexte $=20091106$

29. En France, l'article 1109 du Code civil établit qu' "il n'y a point de consentement valable si le consentement n'a été donné que par erreur ou s'il a été extorqué par violence ou surpris par dol ", ibid.

30. Le droit pose généralement comme condition à la validité du consentement, qu'il soit émis par une personne majeure et possédant des capacités cognitives normales. Pour la France, voir les articles 1123 à 1125-1 du Code civil, ibid.

31. Article 1133, ibid. 
privée $^{32}$, qui est défendue en invoquant le besoin de protéger les individus contre eux-mêmes ${ }^{33}$. L'État interdit le commerce d'organes parce qu'il nuirait à ceux qui le pratiquent, quoi qu'ils en pensent. Notre maxime est ici lue selon l'interprétation b”) : l'activité est essentialisée comme injuria. Il y a là un postulat épistémologique : on suppose que l'État est plus apte à discerner l'intérêt du vendeur que ce dernier.

Ce paternalisme heurte le principe de neutralité de l'État, propre au libéralisme classique de John Stuart Mill par exemple, partisan du laisser-faire. L'auteur parie sur le bon sens du sujet en rejetant tout paternalisme avec le principe de non-nuisance (harm principle), à l'aune duquel la légitimité de l'intervention de l'État dans la sphère privée est évaluée. L'individu possède alors une liberté absolue d'établir n'importe quel contrat, du moment qu'il ne nuit pas à un tiers non-consentant, qu'il est majeur et qu'il possède une rationalité instrumentale. De nombreux libéraux se sont depuis distanciés de cette exigence de neutralité étatique. Rawls en est un exemple, puisqu'il explique dans $A$ Theory of Justice que sous le voile d'ignorance, les personnes de la position originelle choisissent certaines mesures paternalistes ${ }^{34}$. Cette théorie manifeste un souci de protéger les plus faibles contre des décisions qui pourraient leur nuire. Mais du point de vue de la cohérence interne de la doctrine, toute tentative libérale d'intégrer du paternalisme dans une théorie qui se veut avant tout l'étendard de la liberté individuelle se prête à la critique.

Si l'on considère maintenant la pratique du libéralisme, les démocraties dites «libérales" sont en proie à cette même contradiction interne puisqu'elles affirment le droit d'établir un contrat d'une part, et le limitent sévèrement de l'autre. Quel que soit le pays, le législateur qui assure un ordre juridique minimum ${ }^{35}$ a la

32. Le terme paternaliste ne s'applique pas uniquement à l'État, une entreprise peut aussi être paternaliste, comme en témoigne le paternalisme industriel du $19^{\mathrm{e}}$ siècle.

33. On distingue plusieurs types de paternalisme, entre soft et hard, weak et strong. Pour une description complète, voir J. Feinberg, "Legal Paternalism ", Canadian Journal of Philosophy, vol. 1, $\mathrm{n}^{\circ} 1$, septembre 1971, p. 105-124 et Gerald Dworkin, "Paternalism ", The Monist, vol. 56, 1972, p. 64-84. Et pour le hard paternalism, voir Thaddeus Pope, "Counting the Dragon's Teeth and Claws ", Georgia State University Law Review, vol. 20, 2004, p. 659-722.

34. John Rawls, A Theory of Justice, Oxford, Oxford University Press, 1999, par. 15 et 39.

35. Seuls un État de facto inexistant (failed state) ou une anarchie permettraient une réelle indistinction des fins, mais ne seraient pas en mesure de garantir les contrats. 
possibilité d'invalider certains contrats, quand bien même ils satisferaient toutes les conditions normalement requises, y compris le consentement. Si l'on soutient que le consentement est essentiel dans la définition libérale de la liberté individuelle, alors comment une démocratie libérale peut-elle justifier la limitation de la liberté contractuelle?

Selon la thèse "réaliste ", les limites du droit contractuel se posent uniquement en termes d'adéquation avec les mœurs de la majorité. Un contrat est licite tant qu'il ne choque pas l'éthique dominante de la société. Il ne faudrait donc pas s'étonner d'un décalage entre les principes et leur application. Par conséquent, si l'opinion publique estime que vendre un organe est immoral, alors cette interdiction est légitime. Cette thèse est convaincante puisque dans les faits, en Occident, la vente d'organes semble constituer un tabou fortement ancré dans les esprits. Vendre une partie de son corps pour survivre déplaît de toute évidence. On trouve des traces de cette idée dans certaines expressions idiomatiques. En français par exemple, un prix exorbitant représente "les yeux de la tête", en anglais, cela coûte an arm and a leg (" un bras et une jambe "), en espagnol los ojos de la cara ("les yeux du visage»), etc ${ }^{36}$. Ces expressions trahissent une forte aversion à l'idée de devoir vendre une partie de son corps pour survivre. Le dégoût devant le marchandage de Shylock dans Le Marchand de Venise illustre la même idée ${ }^{37}$.

On peut trouver une explication à ce sentiment à travers les racines monothéistes du libéralisme, en vertu desquelles l'homme est conçu comme une créature façonnée à l'image de Dieu. Son corps étant sacré, il ne peut en disposer librement. Il a le devoir de le protéger, puisqu'en dernier ressort son corps ne lui appartient pas. En cohérence avec cette conception, Locke interdit le suicide ${ }^{38}$. Pour justifier cette interdiction, l'auteur explique que si l'individu rationnel souhaite naturellement sa préservation, le droit naturel

36. Rosa Vega Moreno, Creativity and Convention: the Pragmatics of Everyday Figurative Speech, Philadelphie, John Benjamins, 2007 p. 196, n. 11.

37. William Shakespeare, The Merchant of Venice, «(...) let the forfeit be nominated : I for an equal pound of your fair flesh, / to be cut off and taken in what part of your body pleaseth me ", Acte I, scène 3 .

38. John Locke, Two Treatises on Government, éd. par Peter Laslett, Cambridge, Cambridge University Press, 1988, p. 271 ("chacun est tenu (...) de se conserver lui-même et de ne pas abandonner volontairement le milieu où il subsiste »: Deux traités du gouvernement, trad. de l'angl. par Bernard Gilson, Paris, Vrin, 997, p. 141). 
l'exige. Selon cette lecture, si l'être humain est rationnel, et s'il est rationnel de vouloir se préserver, alors il est impossible qu'il puisse consentir à une injuria (hypothèse b'). La liberté individuelle est donc limitée par la préservation de soi. Le tabou autour de la vente de parties de notre corps semble lié à cette idée : vendre un organe serait perçu comme une infraction fondamentale à la préservation de soi, et constituerait par conséquent un acte contraire à la culture issue du monothéisme et à l'anthropologie libérale. Si telle était la perception générale, selon la thèse réaliste, il serait donc justifié d'interdire tout type de contrat pouvant heurter les opinions morales de la majorité.

Cette présomption de la rationalité individuelle propre à l'anthropologie libérale peut donc expliquer la limitation de la liberté contractuelle. Cependant la thèse de la rationalité parfaite est aujourd'hui décriée, y compris par ceux qui fonctionnent à l'intérieur du cadre de l'individualisme méthodologique. C'est le cas par exemple de la thèse de la rationalité limitée émise par l'économiste Herbert Simon. Pour lui, si l'acteur économique a effectivement un comportement rationnel, sa rationalité est limitée en termes de capacité cognitive et de traitement de l'information disponible ${ }^{39}$. Dès lors, il s'arrêterait au premier choix qu'il juge satisfaisant sans considérer toute les options disponibles. Par conséquent, l'individu n'agirait pas toujours dans son "meilleur" intérêt et pourrait consentir à un préjudice. Il pourrait donc convenir dans le cadre d'une théorie de la rationalité limitée de limiter sa liberté contractuelle, pour le protéger.

Dans le débat sur la vente d'organes, cette limitation du droit à établir un contrat n'est pas exactement légitimée de la sorte. Ce n'est pas la rationalité des vendeurs qui est remise en question. On conteste plutôt les circonstances de leur choix. La Déclaration d'Istanbul contre le trafic d'organes et le tourisme de transplantation, qui était une initiative de la Société internationale de transplantation et de la Société internationale de néphrologie, est un bon exemple du discours véhiculé par les législateurs et professionnels concernés par la vente d'organes. Rédigée en mai 2008 par 150 représentants gouvernementaux et responsables médicaux et scientifiques, ce texte définit la vente d'organes de la manière suivante :

39. Herbert Simon, "Theories of Bounded Rationality ", in C. B. Mcguire et Roy Radner, Decision and Organization, Amsterdam, North-Holland Publishing Company, 1972. 
Le trafic d'organes consiste à rechercher (...) des personnes vivantes ou décédées ou leurs organes en faisant usage de menaces, de violence ou de toute autre forme de coercition (...), par la fraude ou par tromperie (...) ou en mettant à profit la vulnérabilité des individus ${ }^{40}$.

La possibilité d'un consentement libre et éclairé du vendeur est exclue. Il semble qu'il faudrait en premier lieu que le vendeur soit parfaitement informé pour que son consentement soit valable. Il doit comprendre tous les risques liés à l'ablation d'un rein. Dans la situation actuelle du marché, ce n'est pas le cas. Selon les études disponibles, la plupart des vendeurs d'organes sont analphabètes ${ }^{41}$. Il leur est donc probablement difficile d'estimer les conséquences de l'ablation d'un organe aussi complexe que le rein. Pire, les ventes sont généralement basées sur le dol: on convainc par exemple des vendeurs ignorants qu'ils seront en meilleure santé après l'opération ${ }^{42}$.

Si en pratique le consentement n'est pas éclairé, la question de principe reste ouverte. Qu'en est-il donc d'un vendeur qui mesurerait parfaitement les conséquences de la vente d'un rein et qui pourtant s'y déciderait ${ }^{43}$ ? Il semble que son consentement serait encore mis en question parce que contraint par la nécessité. En pratique comme en théorie, le vendeur d'organe est conduit à vendre par un besoin impérieux d'argent ${ }^{44}$. Cette réalité est traduite en droit par le concept de "vulnérabilité". La Déclaration d'Istanbul affirme que « le commerce de transplantation devrait être interdit parce qu'il mène inexorablement à l'inégalité et à l'injustice, dans la mesure où il a pour cible des donneurs vulnérables ${ }^{45}$ ". Le concept de vulnérabilité s'applique aussi aux mineurs par exemple,

40. "La déclaration d'Istanbul contre le trafic d'organes et le tourisme de transplantation, Turquie, 30 avril-2 mai 2008 ", Agence de la biomédecine : http://www.agencebiomedecine.fr/article/222.

41. J. Whitehead, "The Harvest: Human Organs and Human Security ", art. cité, p. 24.

42. Par exemple dans N. Schepper-Hughes, "The Ultimate Commodity", art. cité, p. 1349.

43. On recense d'ailleurs quelques cas de vendeurs issus de pays développés, qui sont $a$ priori alphabétisés, ce qui leur permettrait théoriquement de comprendre les conséquences de leur choix, et donc de consentir pleinement.

44. Nous ne souhaitons pas aborder ici le thème de la rémunération par principe des donneurs qui nous semble relever d'une autre question, puisque leur motivation première n'est pas financière, contrairement aux vendeurs d'organes. Sur la rémunération des donneurs, voir V. Gateau, Pour une philosophie du don d'organes, op. cit.

45. "Déclaration d'Istanbul contre le trafic d'organes et le tourisme de transplantation ", op. cit. 
comme cet adolescent chinois qui a vendu son rein pour 2000 euros pour s'acheter un iPad 2, sans le consentement de ses parents ${ }^{46}$. Le droit tient que pour des personnes vulnérables, la vente d'organes ne peut être qu'injuria.

On pourrait rétorquer ici que ce raisonnement suggère que les plus vulnérables ne sont pas en mesure de savoir ce qui est dans leur intérêt. Le consentement serait alors l'apanage des puissants, puisque celui des vulnérables serait immédiatement suspect. Pourtant, ce n'est pas parce qu'elle touche les plus vulnérables que la vente d'organes est interdite. Dans le droit international la vulnérabilité est comprise comme facteur aggravant. La vente d'organes est interdite car elle est essentialisée comme injuria. Mais elle est interdite en particulier aux plus vulnérables car leur consentement est contraint.

Mais pourquoi y aurait-il motif à douter du consentement dès lors que les parties contractantes ne possèderaient pas une connaissance parfaite de leur choix ou seraient contraintes ? C'est la question que pose le philosophe Ruwen Ogien dans L'éthique aujourd'hui. L'auteur dénonce la variabilité des critères de consentement selon les cas. Il prend l'exemple suivant :

Si, pour qu'il soit correct de dire de quelqu'un qu'il a vraiment consenti à une relation sexuelle ou autre, il faut être certain qu'il a formé l'intention préalable de s'engager, qu'il l'a fait sans aucune contrainte du partenaire (...) qu'il possédait une connaissance complète de toutes les étapes et de toutes les conséquences de l'action, (...) alors on ne pourra probablement jamais dire de qui que ce soit qu'il a consenti à quoi que ce soit ${ }^{47}$.

En d'autres termes, si l'on exige trop de l'acteur pour que son consentement soit valable, tout choix devient suspect d'être contraint ou influencé. Pour illustrer cette idée, Ogien reprend une idée formulée par Schumpeter avant lui : "s'il fallait remettre en cause la réalité du consentement des électeurs sous prétexte que leur choix a été "influencé" (...) on ne pourrait plus dire d'aucune élection qu'elle est démocratique ${ }^{48}$ ». En effet, l'électeur, exposé à une

46. "Chine: il vend son rein pour un iPad 2 ", lefigaro.fr du 3 juin 2011 : http://www.lefigaro.fr/flash-actu/2011/06/03/97001-20110603FILWWW00276-chineil-vend-son-rein-pour-un-ipad-2.php.

47. Ruwen Ogien, L'éthique aujourd'hui, maximalistes et minimalistes, Paris, Gallimard, 2007, chap. 10 , p. 180.

48. Ibid., p. 180-181. 
forte propagande médiatique, est nécessairement influencé. Et donc, si l'on suit ce raisonnement, il faut accepter que le consentement soit valide s'il est influencé, au risque d'invalider tout choix électoral. Le résultat de la démonstration nous parait juste. Le vendeur d'organe peut être influencé ou contraint, mais cette contrainte n'est pas une raison suffisante pour invalider son consentement. Si la coercition invalidait le consentement alors il faudrait par exemple aussi invalider le consentement d'un parent à jouer au scrabble après les implorations intempestives de son enfant, ce qui parait absurde. Dans le cas qui nous occupe, la question de la contrainte ressemble de toute façon à un faux problème puisque, la vente d'organes étant essentialisée comme injuria, il est impossible d'y consentir.

Mais pourquoi faudrait-il nécessairement décider de critères de validité du consentement universel en amont de l'objet du consentement? La nature et l'enjeu de son objet sont pourtant forcément pertinents et le consentement peut aussi se penser au cas par cas. La vente d'organes se distingue du vote de bien des manières, mais surtout par son caractère irréversible et grave, dans la mesure où le vendeur devra vivre avec ses conséquences toute sa vie. Ce n'est pas nécessairement le cas du vote car il est possible par exemple de sanctionner un dirigeant, ou encore de ne pas revoter pour la même personne ou le même parti si l'on regrette son choix. Le vote a bien évidemment une incidence sur le bien-être, puisqu'il influe en théorie sur le type de gouvernement d'un pays, mais ses conséquences ne sont pas aussi directement personnelles que celle de l'ablation d'un organe. Pourquoi faudrait-il alors exiger les mêmes critères de consentement dans ces deux cas?

Il paraît donc sensé de poser un principe de proportionnalité dans l'évaluation du consentement. L'irréversibilité de l'acte doit être rapportée au degré de certitude du consentement. Cette conclusion se rapproche d'ailleurs de celle de Mill quand il traite du droit à se vendre comme esclave dans On Liberty. Mill concède au principe de non nuisance cette exception, puisqu'il juge illégitime ce type de contrat. Cela lui semble contradictoire d'exiger au nom de la liberté le droit d'abandonner sa liberté, mais l'auteur est aussi particulièrement préoccupé par le caractère irréversible de cet acte, parce que se vendre comme esclave est un acte par lequel l'acteur abandonne à tout jamais sa liberté ${ }^{49}$.

49. J. S. Mill, On Liberty, New York, Prometheus Books, 1986, p. 117. 
On peut conclure qu'il y a du volenti du côté des vendeurs, même si leur consentement est forcé par les circonstances et que leur information est imparfaite. Pour autant, la nature particulièrement irréversible de la vente d'organes semble poser problème. Mais dans ce cas, c'est nécessairement la question de l'injuria qui est posée, puisqu'ici aussi la vente est essentialisée comme préjudice.

II.

Si ce n'est pas la nature mais l'objet du consentement qui est en question, il convient de se demander si vendre un organe constitue dans l'absolu une injuria. Pour répondre à cette question, il faut en premier lieu savoir si cette opération constitue un préjudice corporel ou psychologique. Dans la littérature sur le sujet, on contourne cette question. La raison en est simple : ceux qui s'insurgent contre ce commerce souhaitent le critiquer sans pour autant écorner le don d'organes, que tous célèbrent comme un geste altruiste. Pourtant, les données empiriques montrent qu'en pratique, les vendeurs souffrent de préjudices physiques importants. Dans un pays développé, il est possible de vivre normalement avec un seul rein, même si les risques de l'opération de prélèvement ne sont pas nuls pour le donneur ${ }^{50}$. De plus, les médecins doivent s'assurer que ce dernier est en parfaite santé lors du prélèvement, sous peine de développer à son tour une insuffisance rénale qui lui serait fatale. Dans un pays en voie de développement, la situation est toute autre. Les médecins malhonnêtes pratiquant les prélèvements ne se préoccupent pas du sort des vendeurs ${ }^{51}$. Les données empiriques montrent que, n'ayant pas accès à des soins gratuits et de bonne qualité ${ }^{52}$, la santé des vendeurs décline, comme le montre une étude parue dans le Journal

50. «Les risques médicaux à court terme sont bien connus et dans l'ensemble très faibles, mais pas nuls. Il s'agit du risque anesthésique, du risque chirurgical d'hémorragie et $\mathrm{du}$ risque postopératoire de thrombose veineuse et d'embolie pulmonaire. Dans la littérature, le risque de mortalité est estimé à $0,03 \%$ ", in Marie-France MamzerBruneel, Catherine Fournier, Christophe Legendre, "La transplantation rénale à partir de donneurs vivants : Enjeux éthiques et juridiques ", Médecine Sciences, n 26, 2010, p. 523.

51. Jayson Rapoport, Alexander Kagan et Michael Friedlaender, "Legalizing the Sale of Kidney Transplantation: Suggested Guidelines ", The Israel Medical Association Journal, vol. 4, décembre 2002, p. 1133.

52. Ibid. 
of the American Medical Association ${ }^{53}$. Sur les 305 vendeurs de la région de Chennai en Inde qui ont été interrogés, la plupart sont des hommes analphabètes travaillant comme journaliers pour des exploitations agricoles. $86 \%$ déclarent avoir vu leur état de santé se détériorer de manière significative, à un tel point que $65 \%$ d'entre eux ont perdu leur emploi pour cause d'incapacité physique. N'ayant pas reçu les soins post-opératoires dont ils avaient besoin, ils ne sont plus en mesure d'exercer de lourds travaux physiques. De plus, comme l'étude de Chennai le montre, après la vente, ils regrettent leur choix et retombent à $79 \%$ dans le cycle de la dette et de la pauvreté. Les vendeurs brésiliens rencontrés par l'anthropologue Nancy Schepper-Hughes parlent quant à eux de douleurs chroniques, de dépression grave et d'un sentiment d'incomplétude post-prélèvement ${ }^{54}$. La cause de ces souffrances est difficile à déterminer, la question est de savoir si l'absence de suivi médical adéquat en est la cause, ou s'ils seraient traumatisés par le fait de vendre une partie d'eux-mêmes. Nous pouvons en tout cas conclure que, dans les conditions actuelles du marché, la vente d'organes inflige aux vendeurs un préjudice physique et psychologique.

Envisageons un scénario utopique dans lequel le vendeur aurait un accès illimité et gratuit à des soins hospitaliers et psychothérapeutiques, et sortirait du cycle de la pauvreté grâce à la vente. Cette situation pourtant idéale ne saurait protéger les vendeurs d'un préjudice que d'aucuns estiment le plus grave de tous, celui de la violation de la dignité humaine. C'est sur ce principe qu'est fondée l'indisponibilité du corps humain dans le droit international. Or, il n'existe pas de définition claire de la dignité humaine. C'est "le plus flou des concepts", pour reprendre les termes d'Olivier Cayla ${ }^{55}$, et pourtant l'argument principal dont on se sert dans tout débat de bioéthique. Il n'en pas toujours été ainsi. En 1948, date de la Déclaration universelle des droits de l'homme, lorsqu'on mentionne dans la première phrase du préambule «la reconnaissance de la dignité inhérente à tous les membres de la famille humaine et de leurs droits égaux et inaliénables (qui) constitue le fondement

53. Madhav Goyal, Ravindra Mehta, Lawrence Schneiderman et al., "Economic and Health Consequences of Selling a Kidney in India ", Journal of the American Medical Association, 2002, vol. 288, no 13, p. 1589-1593.

54. N. Schepper-Hughes, "The Ultimate Commodity ", art. cité, p. 1349.

55. Olivier Cayla, "Dignité humaine : Le plus flou des concepts », Le Monde, 31 janvier 2003. 
de la liberté, de la justice et de la paix dans le monde ${ }^{56}$ ", le monde entier sait parfaitement à quoi il est fait référence. Il s'agit, après la Shoah, d'inscrire dans le droit une parfaite égalité de nature et de droit entre tous.

Le sens partagé de cette dignité s'est détissé au fil du temps. Preuve en est que ce concept est invoqué aujourd'hui de manière contradictoire. Il est souvent utilisé comme principe invalidant le consentement. Dans le cadre du débat sur la vente d'organes, échanger un organe contre de l'argent serait contraire à la dignité inhérente à chaque être. Le consentement ne serait alors pas pertinent dans le débat, parce qu'il s'agit de toute manière d'une violation et donc d'une injuria. Cette position expliquerait l'axiome d'indisponibilité du corps humain : tout produit ou partie du corps humain ne peut être échangé contre de l'argent, car attribuer une valeur monétaire au corps le réifierait. On cite ici généralement Emmanuel Kant, qui aurait dit que «l'homme n'est pas autorisé à vendre un de ses membres pour de l'argent, même s'il devait recevoir dix mille thalers pour un seul de ses doigts ; (...) en contrevenant à cette règle, l'homme se réduit lui-même à l'état de chose ${ }^{57}$ ". Cette même interprétation se retrouve en droit dans le désormais célèbre cas du "lancer de nain " de Morsang-sur-Orge, où le juge du Conseil d'État a expliqué à la personne qui défendait son droit à être lancé contre rémunération, qu'il "nuisait à sa propre dignité $^{58}$ ». Ce cas a été très vivement attaqué comme éminemment paternaliste et discriminatoire. Le juge expliqua que s'il s'était agi de lancers de personnes de taille moyenne, sa décision aurait été autre, les enjeux étant différents. L'exigence de dignité s'appliquerait donc ici exclusivement aux nains, en vertu de leur handicap. Pour Hélène Thomas, cet arrêt distille des relents d'Ancien Régime - l'auteure renvoie à l'origine aristocratique du terme de dignité, dont on se servait pour contraindre les bénéficiaires de charité à un

56. Préambule de la Déclaration universelle des droits de l'homme, disponible en ligne sur le site de l'ONU : http://www.un.org/fr/documents/udhr.

57. Emmanuel Kant, Leçons d'Éthique, trad. de l'all. par Luc Langlois, Paris, Libraire Générale Française, 1997, p. 236.

58. L'argument du "trouble à l'ordre public " a été aussi reçu par le Conseil d'État, mais le cas de la vente d'organes ne présentant pas de manière évidente de lien avec cet argument, nous ne jugeons pas pertinent de développer cette question. Pour plus d'information sur ce cas, voir Arrêté du 27 octobre 1995, Commune de Morsangsur-Orge, Rec. Lebon, p. 372. Pour une perspective critique de l'arrêt, voir : O. Cayla, "Jeux de nains, jeux de vilains", in Gilles Lebreton (dir.), Les droits fondamentaux de la personne humaine en 1995 et 1996, Paris, L'Harmattan, 1998, p. 149-164. 
devoir de décence ${ }^{59}$. Il serait donc possible de dire que la dignité ne peut elle aussi n'être que l'apanage des nantis, puisqu'elle n'est exigée que des plus vulnérables.

Mais depuis quelque temps, la Cour européenne des droits de l'homme s'est emparée du concept pour au contraire donner plus de force au consentement dans des cas de préjudice consenti. Le retentissant cas $\mathrm{KA}$ et $\mathrm{AD}$ c/ Belgique (2005) autorise, au nom de cette même dignité humaine, le droit de pratiquer des violences consenties sadomasochistes, en affirmant un droit absolu à disposer de son corps et au respect de la vie privée. Comme nombre de juristes, le professeur de droit Jean-Pierre Marguénaud dénonce cet arrêt qui «fait voler en éclats le principe d'indisponibilité du corps humain", seul obstacle légal à la commercialisation d'organes humains ${ }^{60}$. La dignité se retournerait alors contre elle-même, en ne protégeant pas les personnes dites vulnérables, mais en présentant une justification pour entériner le consentement des vendeurs, même contraint. Cette nouvelle interprétation du principe de dignité humaine pratiquée par la Cour européenne est foncièrement anti-paternaliste, elle ignore tout du contexte et de l'objet du consentement. En ce sens, elle correspond entièrement à la thèse libertarienne, qui entend la dignité humaine comme exigence d'une absolue autonomie de l'individu.

Cette interprétation de la propriété de soi n'est pas sans difficulté. Invoquée à l'origine par Locke pour justifier l'acquisition de la terre par le travail, la notion de propriété de soi impliquait aussi que l'individu appartient en dernier ressort à son Créateur, comme nous l'avons vu plus haut ${ }^{61}$. Avec la conception libertarienne du libre usage de son corps, on s'éloigne donc du sens premier du concept. De plus, si l'on suit le raisonnement de Quentin Skinner, chez Locke, la propriété de soi ne peut signifier qu'une seule chose : pouvoir jouir librement de ses facultés ${ }^{62}$. En d'autres termes, s'appartenir signifie posséder une totale liberté de mouvement. Par conséquent, seuls l'esclave et l'employé ne sont

59. Hélène Thomas, "Du lancer de nain comme canon de l'indignité. Le fondement éthique de l'État social ", Raisons Politiques, mai 2002, n 6, p. 37-52.

60. Jean-Pierre Marguenaud, «Le contrat portant sur une chose hors commerce : exemple du contrat sur les organes humains ", in Christophe Jamin et Emmanuel Sprinar, Droits et économie des contrats, Paris, LGDJ, 2008, p. 197.

61. J. Locke, Deux traités du gouvernement, op. cit.

62. Quentin Skinner, "Repenser la liberté politique », trad. de l'angl. par Alicia-Dorothy Mornington, Raisons Politiques, vol. 4, $\mathrm{n}^{\circ}$ 36, 2009, p. 111-125. 
pleinement propriétaires d'eux-mêmes, puisque le fruit de leur labeur appartient à leur maître ou à leur supérieur.

Dans Anarchy, State and Utopia, Robert Nozick fournit une application saisissante de cette interprétation de la propriété de soi. Il explique qu'un État de type rawlsien, parce qu'il s'arroge le droit de redistribuer les richesses, pourrait réquisitionner des organes de force. Afin de permettre à tous de jouir du sens de la vue pendant le même nombre d'années, il faudrait redistribuer les cornées ${ }^{63}$. L'exemple volontairement choquant a pour but de faire réfléchir le lecteur au sujet de l'idée même de redistribution, mais il exprime aussi très clairement la lecture nozickéenne de la propriété. Le corps et l'argent sont des possessions au même titre. Il s'agit là d'un dualisme sans ambages et cette conception du corps donne lieu à des conclusions contre-intuitives : un libertarien comprend le viol comme vol, ou comme un empiètement illégitime de la propriété d'autrui ${ }^{64}$. Cette appréhension ne reflète pas le rapport complexe qu'entretient le corps avec l'esprit, ni ce qu'un viol représente comme traumatisme pour la victime.

Admettre qu'un individu est propriétaire de son corps ne résout pas le problème de l'injuria dans la vente d'organes, puisqu'il existe plusieurs manières de comprendre cette propriété. Pour Nozick, ce qui caractérise le concept de propriété, c'est justement l'absolue liberté du propriétaire de décider de l'usage de sa posses$\operatorname{sion}^{65}$. Au contraire, chez Kant, la propriété implique des devoirs envers soi-même : si le corps « appartient au soi (...) notre devoir à son égard consiste d'abord pour notre esprit à le discipliner, et ensuite à en prendre soin ${ }^{66}$ ". Nous avons ici affaire à deux interprétations contradictoires du même terme, et il est difficile de trancher entre la propriété de soi comprise comme droit absolu ou

63. R. Nozick, Anarchy State and Utopia, op. cit., p. 206 : «Maximiser la position des plus démunis pourrait impliquer que l'on redistribue de manière autoritaire les parties du corps ("Vous avez joui du sens de la vue pendant toutes ces années, et maintenant un, voire deux de vos yeux vont être transplantés à d'autres"), ou que l'on tue des personnes en bonne santé pour prélever de leur corps des organes nécessaires à la survie d'autres personnes qui sinon seraient décédées jeunes. ", (notre traduction).

64. Voir à ce titre Alan Wertheimer, Consent to Sexual Relations, Cambridge, Cambridge University Press, 2003, en particulier p. 11-36. Et aussi : Richard Posner, An Economic Analysis of Law, Boston, Little, Brown, 1992 [4 $4^{\mathrm{e}}$ éd.].

65. R. Nozick, Anarchy State and Utopia, op. cit., p. 171 : "L'idée de posséder un droit de propriété sur X (...) signifie avant tout d'avoir le droit de déterminer ce que l'on fait de X. ", (notre traduction).

66. E. Kant, Emmanuel, Leçons d'Éthique, op. cit., p. 282. 
comme entraînant des devoirs envers soi-même. Ces deux interprétations seraient de toute manière absurde si l'on considère que l'on ne saurait être possesseur et possession, comme le défend Michael Sandel ${ }^{67}$. Cet auteur explique que la possession permet de distinguer ce qui est mien de ce qui est sien, mais qu'elle crée aussi une distance entre possession et possesseur puisqu'elle différencie ce qui est mien du moi. Si, pour Nozick, le vendeur n'est pas victime d'injuria, pour Kant il l'est indéniablement. Il semble impossible de conclure par conséquent que les vendeurs souffrent d'une injuria de dignité, puisque le principe de dignité peut donc servir nos lectures b') et b") du principe de volenti non fit injuria. Par sa trop grande ambivalence, le terme de dignité humaine parait désormais difficile à employer.

Quoi qu'il en soit, il faut comprendre que dans la logique libertarienne, cette lecture de la propriété de soi provient d'une peur face à l'expansion possiblement illimitée du pouvoir de l'État. Le libertarien tient à rejeter toute limite extrinsèque à la volonté individuelle puisqu'il pense qu'à partir du moment où l'État interdirait un cas de préjudice consenti, cela créera un précédent et interdire la vente d'organes rendrait tout aussi inévitable une dangereuse montée en puissance de l'intervention étatique dans la vie privée. C'est certainement vrai si l'on considère que tout principe doit être appliqué de manière rigide, et qu'il faut établir des critères fixes et absolus au consentement, en dehors de tout contexte. Mais si l'on décide de traiter le consentement au cas par cas, en considérant dans chaque cas la nature de l'objet et le contexte de l'agent, l'argument de la pente glissante ne se vérifie pas nécessairement.

Pour conclure, dans le cas du marché d'organes, nous avons vu comment le consentement des vendeurs est mis en question, puisqu'en en pratique, il n'est pas informé. Nous avons montré que cet argument est problématique, puisqu'il conduit à invalider la possibilité même du consentement. Nous avons ensuite examiné l'argument de la vulnérabilité, en montrant qu'il sert surtout de circonstance aggravante à la vente d'organes - elle est interdite à tous car elle est essentialisée comme injuria. Nous avons indiqué que la nature irréversible de l'acte pourrait justifier l'adoption de critères de

67. M. Sandel, Libealism and the Limits of Justice, Cambridge, Cambridge University Press, 1998 [ $2^{\text {e }}$ éd.], p. 55. "Dans la mesure où je possède une chose, elle m'est forcément proche et lointaine à la fois (...) cette chose est mienne elle n'est donc pas tienne (...) cette chose est à moi elle n'est donc pas moi. ", (notre traduction). 
validité du consentement plus ou moins souples. Cela nous a conduits à nous pencher sur la réalité du préjudice, qui se vérifie dans les faits lorsqu'il s'agit de préjudice physique et psychologique. Il est peu aisé de savoir si la vente atteint la dignité des vendeurs puisque ce terme est trop équivoque. Finalement, notre analyse de la maxime volenti non fit injuria montre que si le consentement est essentiel, son objet ne doit pas pour autant être ignoré.

Alicia-Dorothy Mornington est doctorante en pensée politique à Sciences Po (Paris) où elle y enseigne la théorie politique. Elle travaille sur la notion de préjudice consenti, ce qui l'a conduite à s'intéresser à l'articulation entre théorie et pratique du libéralisme, en mettant en rapport la littérature libérale et la jurisprudence contemporaine de cas de préjudice consenti. Elle est l'auteure de "Raymond Bellour : Le Corps du cinéma : Hypnoses, émotions, animalités " (Raisons politiques, $\mathrm{n}^{\circ} 38$, juin 2010) et de la traduction de l'article de Quentin Skinner : « Repenser la Liberté Politique»(Raisons politiques, $\mathrm{n}^{\circ} 36$, janvier 2010).

\section{RÉSUMÉ}

Vendre ses organes : un cas de préjudice consenti ?

Au cœur du débat sur la légalisation de la vente d'organe figure un individu qui consent à vendre une partie de son corps. Ce cas permet de poser la question du préjudice consenti puisque le vendeur semble consentir à un acte qui peut paraitre préjudiciable. Â l'aune de la maxime romaine volenti non fit injuria, cet article commence par déplier la question du consentement, en mettant en relief la structure argumentative permettant d'invalider celui du vendeur. L'analyse se focalise sur la notion d'information asymétrique, de contrainte et de vulnérabilité. Puis l'auteure considère la nature du préjudice subi par le vendeur, en examinant le préjudice corporel et psychologique ainsi que la question de la dignité humaine.

\section{Is selling organs consent to harm?}

At the premise of the debate around organ sale is an individual consenting to sell a body part. This case allows us to analyse the issue of consent to harm because the seller seems to consent to something which seems to qualify as "harm". By using the volenti non fit injuria doctrine, the article starts by examining the seller's consent and arguments used to invalidate it, such as asymmetric information, coercion and vulnerability. It then goes on by looking at the question of harm, looking at bodily and psychological harm and lastly at the notion of human dignity. 